\title{
Report on the second Mock LISA data challenge
}

\author{
Stanislav Babak ${ }^{1}$, John G Baker ${ }^{2}$, Matthew J Benacquista ${ }^{3}$, \\ Neil J Cornish ${ }^{4}$, Jeff Crowder ${ }^{5}$, Curt Cutler ${ }^{5,6}$, Shane L Larson ${ }^{7}$, \\ Tyson B Littenberg ${ }^{4}$, Edward K Porter ${ }^{1}$, Michele Vallisneri ${ }^{5,6}$, \\ Alberto Vecchio $^{8,9}$ (the Mock LISA data challenge task force), \\ Gerard Auger $^{10}$, Leor Barack ${ }^{11}$, Arkadiusz Błaut ${ }^{12}$, Ed Bloomer $^{13}$, \\ Duncan A Brown ${ }^{6,14,15}$, Nelson Christensen ${ }^{16}$, James Clark ${ }^{13}$, \\ Stephen Fairhurst ${ }^{6,15,17}$, Jonathan R Gair ${ }^{18}$, Hubert Halloin ${ }^{10}$, \\ Martin Hendry $^{13}$, Arturo Jimenez ${ }^{3}$, Andrzej Królak ${ }^{19}$, Ilya Mandel ${ }^{6,9}$, \\ Chris Messenger ${ }^{13}$, Renate Meyer ${ }^{20}$, Soumya Mohanty ${ }^{3}$, Rajesh Nayak ${ }^{3}$, \\ Antoine Petiteau ${ }^{10}$, Matt Pitkin ${ }^{13}$, Eric Plagnol ${ }^{10}$, Reinhard Prix ${ }^{1}$, \\ Emma L Robinson ${ }^{8}$, Christian Roever ${ }^{20}$, Pavlin Savov ${ }^{6}$, Alexander \\ Stroeer $^{8,9}$, Jennifer Toher ${ }^{13}$, John Veitch ${ }^{8}$, Jean-Yves Vinet ${ }^{21}$, \\ Linqing Wen ${ }^{1}$, John T Whelan ${ }^{1}$ and Graham Woan ${ }^{13}$ (the Challenge-2 \\ participants)
}

${ }^{1}$ Max-Planck-Institut für Gravitationsphysik (Albert-Einstein-Institut), Am Mühlenberg 1, D-14476 Golm bei Potsdam, Germany

2 Gravitational Astrophysics Laboratory, NASA Goddard Space Flight Center, 8800 Greenbelt Rd., Greenbelt, MD 20771, USA

${ }^{3}$ Center for Gravitational Wave Astronomy, University of Texas at Brownsville, Brownsville, TX 78520, USA

${ }^{4}$ Department of Physics, Montana State University, Bozeman, MT 59717, USA

5 Jet Propulsion Laboratory, California Institute of Technology, Pasadena, CA 91109, USA

${ }^{6}$ Theoretical Astrophysics, California Institute of Technology, Pasadena, CA 91125, USA

${ }^{7}$ Department of Physics, Weber State University, 2508 University Circle, Ogden, UT 84408, USA

${ }^{8}$ School of Physics and Astronomy, University of Birmingham, Edgbaston, Birmingham B152TT, UK

${ }^{9}$ Department of Physics and Astronomy, Northwestern University, Evanston, IL, USA

${ }^{10}$ APC, UMR 7164, University Paris 7 Denis Diderot, 10, rue Alice Domon et Leonie Duquet, 75025 Paris Cedex 13, France

${ }^{11}$ School of Mathematics, University of Southampton, Southampton SO171BJ, UK

12 Institute of Theoretical Physics, University of Wrocław, Wrocław, Poland

13 Department of Physics and Astronomy, University of Glasgow, Glasgow, UK

${ }^{14}$ Department of Physics, Syracuse University, Syracuse NY13244, USA

${ }^{15}$ LIGO Laboratory, California Institute of Technology, Pasadena, CA 91125, USA

${ }^{16}$ Physics and Astronomy, Carleton College, Northfield, MN, USA

${ }^{17}$ School of Physics and Astronomy, Cardiff University, 5, TheParade, Cardiff CF243YB, UK

${ }^{18}$ Institute of Astronomy, University of Cambridge, Cambridge CB30HA, UK

${ }^{19}$ Institute of Mathematics, Polish Academy of Sciences, Warsaw, Poland

${ }^{20}$ Department of Statistics, The University of Auckland, Auckland, New Zealand

${ }^{21}$ ARTEMIS, Observatoire de la Cote d'Azur-C.N.R.S., 06304 Nice, France

E-mail: Michele.Vallisneri@jpl.nasa.gov

Received 11 November 2007

Published 15 May 2008

Online at stacks.iop.org/CQG/25/114037 


\begin{abstract}
The Mock LISA data challenges are a program to demonstrate LISA dataanalysis capabilities and to encourage their development. Each round of challenges consists of several data sets containing simulated instrument noise and gravitational waves from sources of undisclosed parameters. Participants are asked to analyze the data sets and report the maximum information about the source parameters. The challenges are being released in rounds of increasing complexity and realism: here we present the results of Challenge 2, issued in Jan 2007, which successfully demonstrated the recovery of signals from nonspinning supermassive-black-hole binaries with optimal SNRs between $\sim 10$ and 2000, from $\sim 20000$ overlapping galactic white-dwarf binaries (among a realistically distributed population of 26 million), and from the extreme-massratio inspirals of compact objects into central galactic black holes with optimal SNRs $\sim 100$.
\end{abstract}

PACS numbers: $04.80 . \mathrm{Nn}, 95.55 . \mathrm{Ym}$

(Some figures in this article are in colour only in the electronic version)

\title{
1. Introduction
}

The laser interferometer space antenna (LISA), a NASA and ESA space mission to detect gravitational waves $(\mathrm{GWs})$ in the $10^{-5}-10^{-1} \mathrm{~Hz}$ range [1], will produce time series consisting of the superposition of the signals from millions of sources, many in our galaxy, some as far as the edge of the observable universe. Some of the signals, such as those from extreme-massratio inspirals (EMRIs), are very complex functions of the source parameters; others, such as those from galactic white-dwarf binaries, are simpler, but their resolution will be confused by the presence of many other similar signals that overlap in frequency space. Thus, data analysis is integral to the LISA measurement concept, because no source can be observed without first carefully teasing out its individual voice in the noisy party of the LISA data. Indeed, it is important to understand LISA data analysis in order to demonstrate that the mission can meet its science requirements, and to translate these into decisions on instrument design.

The idea of the Mock LISA data challenges (MLDCs) arose in late 2005 from this very realization. The MLDCs have the purpose of encouraging and tracking progress in LISA data-analysis development, and (as a useful byproduct) of producing a prototype of the LISA computational infrastructure, including common data formats, standard models of the LISA orbits, noises and measurements, software tools to generate waveforms and to simulate the LISA response and more. The MLDCs are a coordinated (but voluntary) effort in the GW community, whereby a task force chartered by the LISA international science team periodically issues data sets containing synthetic noise and GW signals from sources of undisclosed parameters; challenge participants return detection candidates and parameter estimates, together with descriptions of their search methods. These results are then compiled and compared to the previously secret challenge 'key'.

Challenge 1, issued in Jun 2006 with results due in Dec 2006 (see [2, 3]), tackled the detection and parameter characterization of verification binaries (galactic binaries of known frequency and position); of loud unknown galactic binaries, either alone or in small, moderately interfering groups; and of relatively loud inspirals of nonspinning supermassive-black-hole 
$(\mathrm{MBH})$ binaries. All sources were represented by somewhat idealized waveforms, and they were staged on simulated instrument noise alone. Ten collaborations submitted entries, adopting a variety of methods (template-bank, stochastic- and genetic-optimization matched filtering; time-frequency; tomography; Hilbert transform). Despite the short timescale, each challenge was 'solved' by at least one group, although some searches locked on strong secondary probability maxima for the source parameters. More important, Challenge 1 helped set the playing field and assemble the computational tools for the more realistic Challenge 2 .

Challenge 2, issued in Jan 2007 with results due at the end of Jun 2007, raised the bar by proposing three complex subchallenges. Data set 2.1 contained signals from a full population of galactic binary systems (about 26 million sources). Data set 2.2 contained signals from a similar (but distinct) galactic-binary population, plus an undisclosed number (between 4 and 6) of signals from nonspinning-MBH binary inspirals with optimal single-interferometer signal-to-noise ratios (SNRs) between 10 and 2000 and a variety of coalescence times, and plus five EMRI signals with optimal SNRs between 30 and 100. The EMRIs were modeled as Barack and Cutler's 'analytic kludges' [4]: adiabatic sequences of elliptical orbits emitting Peters-Mathews waveforms, with separation, precession and eccentricity evolving according to post-Newtonian equations. Last, five more data sets (denoted 1.3.1-5, since they were released at the time of Challenge 1) contained single EMRI signals over instrument noise alone, with optimal SNRs between 40 and 110. See [5] for more details about the signal models and the ranges from which the source parameters were drawn.

Thirteen collaborations (comprising all the researchers listed as participants in the byline of this article, and most task force members) submitted a total of 22 entries, including a proof-of-principle analysis for stochastic backgrounds performed on data set 2.1. Altogether, Challenge 2 successfully demonstrated the identification of $\sim 20000$ galactic binaries, the accurate estimation of nonspinning-MBH inspiral parameters, and the positive detection of EMRIs, as we discuss in more detail in the rest of this paper. All the solutions submitted by participating groups, together with technical write-ups of their methods and findings, can be found at the URL www.tapir.caltech.edu/mldc/results2. A few groups are also contributing descriptions of their work to the proceedings of this conference.

\section{Data sets 2.1 and 2.2: the galaxy}

Five groups submitted galactic-binary catalogs for data sets 2.1 and 2.2.

GLIG. This collaboration of research groups at institutions in the UK, USA and New Zealand developed a reversible-jump Markov chain Monte Carlo (RJ MCMC) code that can sample models with different numbers of sources; due to lack of time, however, they only submitted parameter sets for the verification binaries.

IMPAN. Królak and Błaut developed an $\mathcal{F}$-statistic, template-bank-based matched-filtering search $[6,7]$ and submitted parameters for 404 sources for data set 2.1.

MTJPL. The Montana state-JPL collaboration used a Metropolis-Hastings Monte Carlo (MHMC) code that ran separately for overlapping frequency bands and for different hypothesized numbers of sources; model comparison was then used to determine the most probable number of sources in each band. MTJPL submitted parameter sets for 19324 sources for data set 2.1, and 18461 sources for 2.2.

PrixWhelanAEI. Prix and Whelan developed an $\mathcal{F}$-statistic, template-bank-based matchedfiltering search using a hierarchical scheme that enforced trigger coincidence between TDI 

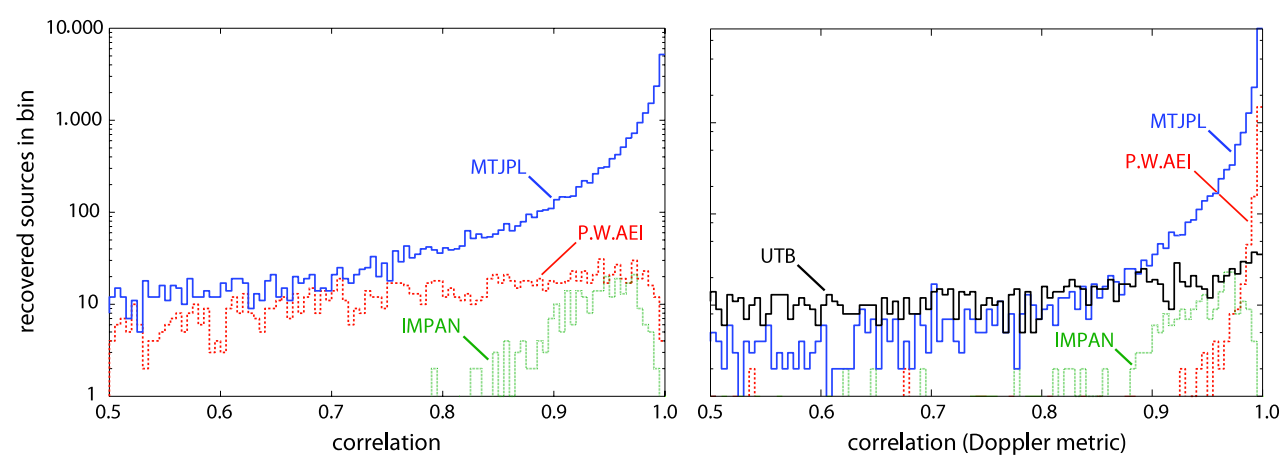

Figure 1. Signal-correlation analysis of Challenge-2.1 galactic-binary catalogs (histogram, with bin fractions on the logarithmic scale). Left panel: reported and injected sources associated by correlation; right: associated by Doppler metric.

observables, followed by a coherent follow-up using noise-orthogonal TDI combinations [8]. They submitted parameter sets for 1777 sources for data set 2.1, and 1737 sources for 2.2.

UTB. Nayak, Jimenez and Mohanty used a tomographic reconstruction technique and submitted parameters for 3862 sources in data set 2.1 .

Evaluation of the performance of these searches brings up several problems of principle: while we know that many of the $\sim 30$ million galactic sources that were injected into the data sets cannot be recovered because they are (relatively) too weak, we do not have a precise estimate of how many sources should be recoverable. Thus, the notion of false dismissal is not well defined. To make matters worse, the notion of false positive is also ill defined, because a single recovered source can provide a good fit to the blended signal from several injected sources, which may well be the 'right' answer with the knowledge we have, since it is the best fit to the data with the smallest numbers of parameters.

The task force devoted considerable time to the analysis of galactic-binary searches, and we do not have space here to describe all the treatments that we applied to the data. Instead, we will limit our report to techniques that pair up individual recovered sources with individual sources from the challenge key, with the understanding that this will overestimate the number of false positives, and say nothing about false dismissals.

One way to proceed is to associate the reported and injected sources that have the strongest signal correlation (in terms of the noise-weighted inner product between the injected and recovered sources), limiting the search to the bright injected sources (with optimal SNR > 2) that could in principle have been found: in the left panel of figure 1 we show the distribution of correlations generated with this procedure for data set 2.1. Detections with the highest correlations can be considered reliable, while those with the lowest correlations probably represent spurious associations.

Another procedure is to associate the reported and injected sources that minimize the Doppler distance that spans the frequency-sky-location subspace of the full parameter space, and automatically maximizes correlation over the extrinsic parameters (amplitude, polarization, inclination, initial phase): the right panel of figure 1 shows the resulting distribution of correlations. The UTB entry, which includes frequency and sky position but not the extrinsic parameters, can only be plotted this way. Generally, this is a softer criterion, and all searches yield better correlations by this measure (especially the PrixWhelanAEI entry, whose long-wavelength approximation for the LISA response is prone to extrinsic-parameter errors). 

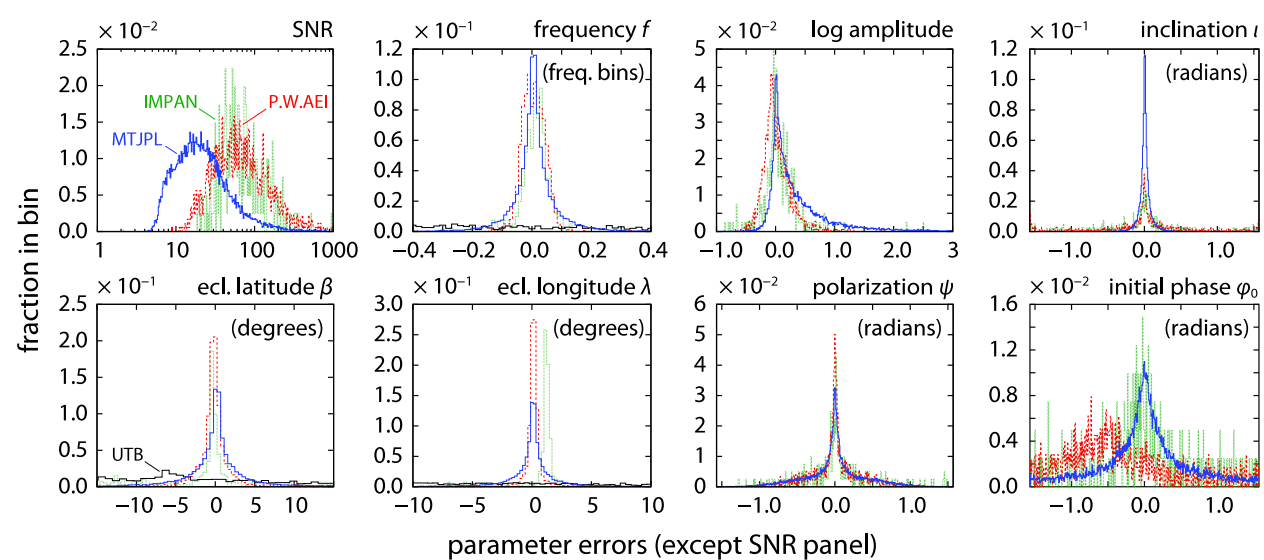

Figure 2. Recovered SNRs and intrinsic and extrinsic parameter errors for Challenge-2.1 galacticbinary catalogs (histogram). True sources and templates are associated by correlation, except for the UTB catalog, for which they are associated by Doppler metric. See [5] for details on the assumed galactic-binary waveforms.

Figure 2 shows the SNRs of the recovered sources and the errors for the intrinsic and extrinsic parameters, computed after associating sources by correlation (and by Doppler metric only for the UTB entry), again for data set 2.1. The errors in frequency are in most cases within a small fraction of a Fourier bin, and the errors in sky position are within a few degrees; by contrast, the errors in the amplitude and in the (extrinsic) orientation angles are larger. The $\phi_{0}$ graph for the PrixWhelanAEI entry suggests a systematic error in the definition of initial phase.

Altogether, these challenges demonstrated a solid capability in analyzing signals from the galaxy and resolving a large number of binaries. As we mentioned, deciding how well they were recovered is not an easy question to answer, because of the difficulty of defining (at least operationally) a notion of identity for recovered sources. These problems deserve careful attention in the future.

\section{Data set 2.2: MBH binaries (over the galaxy)}

Four groups reported parameter sets for the MBH binaries in data set 2.2:

AEIse. Babak and Porter used an $\mathcal{F}$-statistic, template-bank-based matched-filtering search, followed by an MCMC stage.

MTAEI. Cornish and Porter used an MHMC matched-filtering search with a frequencyannealed scheme where shorter, lower-frequency templates are used in the initial phases of the search and then progressively extended [9].

JPLCT. The JPL-CIT collaboration used a three-stage pipeline consisting of a track search in the time-frequency (TF) plane, followed by template-bank-based matched filtering, and by an MCMC refinement [10].

LisaFrance. The French collaboration used a TF track search alone, and therefore reported only mass and time-of-coalescence parameters.

All four MBH binaries in the data set (MBH-1, 2, 4 and 5, with total optimal SNRs $\sim 2583,25,174$ and 117) were positively detected by AEIse, MTAEI, and JPLCT; the TF 
Table 1. Recovered SNRs and parameter errors for MBH-1. Here $M_{c} \equiv\left(m_{1} m_{2}\right)^{3 / 5} /\left(m_{1}+m_{2}\right)^{1 / 5}$ and $\mu=m_{1} m_{2} /\left(m_{1}+m_{2}\right)$ are the chirp and reduced masses; $t_{c}$ and $\phi_{c}$ are the time and GW phase at coalescence; $\beta$ and $\lambda$ are the ecliptic latitude and longitude; $D$ is the luminosity distance; $\iota$ and $\psi$ are the inclination and polarization angles (see [5] for details). All errors on angles are given in radians; the true (optimal) SNR is $\mathbf{2 5 8 3 . 4 2 .}$

\begin{tabular}{|c|c|c|c|c|c|c|c|c|c|c|}
\hline & SNR & $\begin{array}{l}\Delta M_{c} / M_{c} \\
\times 10^{-5}\end{array}$ & $\begin{array}{l}\Delta \mu / \mu \\
\times 10^{-5}\end{array}$ & $\begin{array}{l}\Delta t_{c} / t_{c} \\
\times 10^{-6}\end{array}$ & $\begin{array}{l}\Delta \beta \\
\times 10^{-1}\end{array}$ & $\begin{array}{l}\Delta \lambda \\
\times 10^{-2}\end{array}$ & $\begin{array}{l}\Delta D / D \\
\times 10^{-2}\end{array}$ & $\begin{array}{l}\Delta \iota \\
\times 10^{-2}\end{array}$ & $\begin{array}{l}\Delta \psi \\
\times 10^{-1}\end{array}$ & $\begin{array}{l}\Delta \phi_{c} \\
\times 10^{-1}\end{array}$ \\
\hline AEIse & 2247.60 & 147.0 & 3386.2 & 19.0 & -5.07 & -82.1 & 77.8 & -6.86 & 13.8 & -6.82 \\
\hline MTAEI & 2583.34 & 7.9 & 9.1 & 3.6 & 1.65 & -1.2 & 3.5 & 4.94 & 1.2 & -7.70 \\
\hline JPLCT & 2582.42 & 27.5 & 28.7 & 16.0 & 4.81 & 12.2 & 12.3 & -3.19 & -12.1 & 7.45 \\
\hline lisaFrance & & 2944.6 & 72.7 & 67.8 & & & & & & \\
\hline
\end{tabular}

Table 2. Recovered SNRs and parameter errors for MBH-4, given as in table 1. The true (optimal) SNR is $\mathbf{1 7 4 . 1 2}$.

\begin{tabular}{lrrrrrrrrrr}
\hline & & \multicolumn{1}{c}{$\Delta M_{c} / M_{c}$} & $\Delta \mu / \mu$ & $\Delta t_{c} / t_{c}$ & \multicolumn{1}{c}{$\Delta \beta$} & \multicolumn{1}{c}{$\Delta \lambda$} & $\Delta D / D$ & \multicolumn{1}{c}{$\Delta$} & \multicolumn{1}{c}{$\Delta \psi$} & \multicolumn{1}{c}{$\Delta \phi_{c}$} \\
& \multicolumn{1}{c}{$\mathrm{SNR}$} & $\times 10^{-6}$ & $\times 10^{-4}$ & $\times 10^{-6}$ & $\times 10^{-2}$ & $\times 10^{-2}$ & $\times 10^{-3}$ & $\times 10^{-3}$ & $\times 10^{-3}$ & $\times 10^{-1}$ \\
\hline AEIse & 81.38 & 1396.3 & 149.9 & 3.4 & -12.5 & 104.7 & 574.0 & 3.5 & -185.4 & 8.1 \\
MTAEI & 174.13 & 148.8 & 21.3 & 2.1 & 2.4 & 2.1 & 15.1 & 2.8 & -7.5 & 1.7 \\
& 174.11 & 17.1 & 20.5 & 33.3 & -42.4 & -310.6 & 16.7 & -13.7 & -146.3 & -6.3 \\
JPLCT & 174.11 & 4.2 & 9.5 & 2.1 & 7.8 & 9.6 & 1.2 & 1.3 & -21.3 & -5.4 \\
& 174.12 & 124.7 & 9.0 & 35.4 & -47.3 & -302.9 & 6.3 & -12.4 & 1436.4 & -12.4 \\
\multirow{2}{*}{ lisaFrance } & & 34394.1 & 1804.1 & 280.8 & & & & & & \\
\hline
\end{tabular}

method used by LisaFrance identified MBH-1 and MBH-4, but not MBH-2, and could report only a time of coalescence for MBH-5.

Tables 1 and 2 show fractional parameter errors for MBH- 1 and MBH-4, together with the SNR recovered by the best-fit candidates, computed as

$$
\mathrm{SNR}_{\text {best }}=\frac{\left(A_{\text {true }} \mid A_{\text {best }}\right)+\left(E_{\text {true }} \mid E_{\text {best }}\right)}{\sqrt{\left(A_{\text {best }} \mid A_{\text {best }}\right)+\left(E_{\text {best }} \mid E_{\text {best }}\right)}},
$$

with $(\cdot \cdot)$ being the usual noise-weighted inner product, and $A=(2 X-Y-Z) / 3$ and $E=(Z-Y) / \sqrt{3}$ are the two noise-orthogonal TDI observables (see, e.g., [11]). Table 1 shows that the JPLCT search for MBH-1 locked onto a secondary probability maximum with $\mathrm{SNR}_{\text {best }}$ only slightly lower than the optimal value, but with sky positions off by several degrees, which also led to errors in the other parameters. The JPLCT authors conjecture that this was caused by first subtracting a rough MBH- 1 model from the data, then subtracting the resolvable galactic binaries, and finally refining the MBH search.

MBH-4 (table 1) is an interesting example of a 'true' bimodal probability distribution for the source parameters. MTAEI and JPLCT each submitted two candidates, placed at rather different sky locations, quoting relative probability ratios of $1: 1$ and 1.18:1. In this case, it was probably the sky position and orientation of this source that conspired to degrade LISA's positional sensitivity, since they resulted in a very weak signal in one of the noise-orthogonal observables.

Altogether, this challenge demonstrated a solid capability in the detection and parameter estimation of nonspinning-MBH inspirals with moderate optimal SNRs, even in the presence of a strong galactic background, at least if the inspirals can be considered close to our idealized model: circular and adiabatic with negligible spin effects. These restrictions are being relaxed for the upcoming Challenge 3 . 
Table 3. Recovered SNRs and parameter errors for the EMRI signal in data set 1.3.1. Here $\beta$ and $\lambda$ are the ecliptic latitude and longitude; $\theta_{K}, \phi_{K}$, and $a$ set the orientation and magnitude of the central-BH spin; $\mu$ and $M$ are the compact-object and central-BH masses; $\nu_{0}$ is the initial radial orbital frequency; $e_{0}$ is the initial eccentricity; and $D$ is the luminosity distance. All errors are given as fractions of the allowed prior range for the corresponding parameters $\left(0.15\right.$ for $\left.e_{0}\right)$, except for $v_{0}$ and $D$. Not all parameters are shown. See [5] for details on the assumed EMRI waveforms. The true (optimal) SNR is $\mathbf{1 3 0 . 9 8}$.

\begin{tabular}{llllllllllll}
\hline & SNR & $\delta$ & $\delta \lambda$ & $\delta \theta_{K}$ & $\delta \phi_{K}$ & $\delta a$ & $\delta \mu$ & $\delta M$ & $\frac{\Delta v_{0}}{\nu_{0}}$ & $\delta e_{0}$ & $\frac{\Delta D}{D}$ \\
\hline BBGP & 74.86 & -0.33 & -0.0095 & -0.13 & -0.076 & 0.28 & -0.15 & -0.51 & 0.017 & 0.21 & -1.21 \\
& 72.96 & -0.32 & 0.011 & -0.15 & -0.078 & 0.27 & -0.15 & -0.51 & 0.017 & 0.21 & -1.22 \\
& 72.52 & -0.28 & 0.025 & -0.063 & -0.036 & 0.41 & -0.17 & -0.35 & -0.009 & 0.29 & -2.15 \\
& 72.49 & -0.28 & 0.025 & -0.063 & -0.034 & 0.41 & -0.17 & -0.36 & -0.009 & 0.29 & -2.17 \\
& 70.59 & -0.31 & -0.020 & -0.36 & -0.21 & 0.44 & -0.12 & -0.12 & -0.03 & 0.28 & -0.91 \\
EtfAG & & 0.016 & 0.0012 & & & -0.082 & 0.10 & -0.17 & 0.0026 & 0.098 & \\
MT & 74.85 & 0.15 & 0.47 & -0.069 & -0.15 & -0.026 & 0.073 & 0.18 & 0.00025 & -0.11 & -0.71 \\
& 76.52 & 0.084 & -0.49 & -0.33 & -0.10 & -0.022 & 0.046 & 0.16 & 0.00026 & -0.10 & -0.70 \\
\hline
\end{tabular}

\section{Data sets 1.3.X: EMRIs}

Three groups reported parameter sets for the EMRIs in data sets 1.3.1-1.3.4 (but none for 1.3.5). No group tackled the problem of detecting these systems in data set 2.2 (on top of the galactic background).

$B B G P$. Babak and colleagues used an MCMC matched-filtering search that modeled the signal with a sequence of progressively longer templates (a time-annealed scheme).

EtfAG. Gair, Mandel and Wen used a TF track search that (for now) targeted only the intrinsic parameters and sky position [12].

MT. Cornish used an MHMC matched-filtering search, running it in parallel on individual month-long segments that were subsequently strung together.

Table 3 shows typical recovered SNRs and errors. A comparison of the optimal and recovered SNRs indicates that the matched-filtering searches locked on several secondary probability maxima with similar probabilities. Still, the recovered SNRs correspond to solid detections with exceedingly low false-alarm probabilities. The errors are quoted as fractions of the allowed parameter ranges, and they are quite large. Intriguingly, the TF search was most accurate in determining the sky position.

Altogether, these challenges demonstrated a positive capability of detecting EMRIs, at least if their signals are similar in complexity to the kludge waveforms used in this challenge [5]; however, the prospects for accurate parameter estimation are still uncertain, and a good focus for further challenges.

\section{Conclusion}

We are very excited about the outcome of the first two MLDCs, which have given a convincing demonstration that a significant portion of the LISA science objectives could already be achieved with techniques that are currently in hand. Most of the research groups that participated in Challenge 1 have successfully made the transition to the greater complexity of Challenge 2. Challenge 3 (with data sets released in Jan 2008 and results due in Dec 2008) 
will continue to move in the direction of more realistic signals, featuring chirping galactic binaries and precessing binaries of spinning MBHs. It will also include two new classes of signals: an isotropic primordial GW background and bursts from the cusps of cosmic strings. In addition, Challenge 1B took place between July and Dec 2007. This was a repeat of Challenge 1, conceived to provide a softer entry point for research groups new to the MLDCs. Ten collaborations (including five new institutions) participated, demonstrating increasing sophistication and proficiency in a range of LISA data-analysis techniques.

Furthermore, the MLDC conventions, file formats and software tools (see lisatools.googlecode.com) have matured to the point where interested parties can use them to generate a variety of data sets. This enables a wealth of interesting side investigations, such as the studies of the LISA science reach that are now being undertaken by the LISA science team. To obtain more information and to participate in the MLDCs, see the official MLDC website (astrogravs.nasa.gov/docs/mldc) and the task force wiki (www.tapir.caltech.edu/listwg1b).

\section{Acknowledgments}

SB, EKP and JTW acknowledge support from the German Aerospace Center (DLR) and the Max-Planck Society. MB: from NASA grant NNG04GD52G and the NASA Center for GW Astronomy at the University of Texas, Brownsville (NAG5-13396). NC: NASA grants NNG05GI69G and NNX07AJ61G. MV: the LISA Mission Science Office and by JPL's HRDF. DB and SF: NSF grant PHY-0601459 and the LIGO Laboratory JG: St Catharine's College, Cambridge. IM: the Brinson Foundation, NASA grant NNG04GK98G and NSF grant PHY0601459. RP: the Max-Planck Society. LW: the Alexander von Humboldt Foundation's Sofja Kovalevskaja program, funded by the German Federal Ministry of Education and Research. JC's, CC's and MV's work was carried out at the Jet Propulsion laboratory, California Institute of Technology, under contract with the National Aeronautics and Space Administration.

\section{References}

[1] Bender P and Danzmann P 1998 (the LISA Study Team) Laser Interferometer Space Antenna for the Detection of Gravitational Waves, Pre-Phase A Report vol MPQ 233 (Garching: Max-Planck-Institüt für Quantenoptik)

[2] Arnaud K A et al 2006 (the MLDC Task Force) Laser Interferometer Space Antenna 6th Int. LISA Symp. (Greenbelt, MD, 19-23 June 2006) ed S M Merkowitz and J C Livas (Melville, NY: AIP) p 619

Arnaud K A et al 2006 (the MLDC Task Force) Laser Interferometer Space Antenna 6th Int. LISA Symp. (Greenbelt, MD, 19-23 June 2006) ed S M Merkowitz and J C Livas (Melville, NY: AIP) p 625

[3] Arnaud K A et al 2007 (the MLDC Task Force and Challenge 1 participants) Class. Quantum Grav. 24 S529

[4] Barack L and Cutler C 2004 Phys. Rev. D 69082005

[5] Arnaud K A et al 2007 (the MLDC Task Force) Class. Quantum Grav. 24 S551

[6] Jaranowski P, Królak A and Schutz B F 1998 Phys. Rev. D 58063001

[7] Królak A, Tinto M and Vallisneri M 2004 Phys. Rev. D 70022003

[8] Prix R and Whelan J T 2007 Class. Quantum Grav. 24 S565 Poster www.ligo.caltech.edu/docs/G /G070462-00.pdf

[9] Cornish N J and Porter E K 2007 Class. Quantum Grav. 24 5729-55

[10] Brown D A, Crowder J, Cutler C, Mandel I and Vallisneri M 2007 Class. Quantum Grav. 24 S595

[11] Vallisneri M, Crowder J and Tinto M 2007 Sensitivity and parameter-estimation precision for alternate LISA configurations Preprint 0710.4369

[12] Gair J R, Mandel I and Wen L 2007 Proc. 7th Amaldi Conf. on Gravitational Waves (Sydney, 8-14 July 2007) (Preprint 0710.5250) (submitted) 